03

\title{
Генерация суммарной частоты от тонкого сферического слоя. I. Аналитическое решение
}

\author{
(C) В.Н. Капшай, А.А. Шамына \\ Гомельский государственный университет им. Ф. Скорины, \\ 246019 Гомель, Беларусь \\ e-mail: anton.shamyna@gmail.com, kapshai@rambler.ru
}

Поступила в редакцию 22.02.2018 г.

В тензорном виде в обобщенном приближении Релея-Ганса-Дебая решена задача о генерации гармоники суммарной частоты от тонкого нелинейного слоя, нанесенного на диэлектрическую сферическую частицу, помещенную в диэлектрическую среду. Тензор нелинейной диэлектрической восприимчивости второго порядка выбран в общей форме, содержащей киральные компоненты. В векторной и тензорной формах получены выражения, описывающие пространственное распределение поля излучения суммарной частоты, генерируемого двумя плоскими электромагнитными эллиптически поляризованными волнами. Получены предельные выражения, описывающие пространственное распределение гармоники суммарной частоты при малых и больших радиусах сферического слоя. Обнаружено, что при малых радиусах сферического слоя излучение, обусловленное киральными коэффициентами анизотропии, вносит доминирующий вклад в генерацию.

DOI: $10.21883 / O S .2018 .06 .46083 .55-18$

\section{Введение}

Дипольная модель, используемая для описания нелинейных оптических явлений, запрещает нелинейные эффекты второго порядка в центросимметричных средах. Это ограничение снимается при рассмотрении эффектов на поверхности частиц, где условие центросимметричности нарушается [1]. Преимушеством основанного на этом метода исследования поверхностей частиц перед другими является его избирательность, так как. отсутствует сигнал суммарной частоты от их объема (если они расположены хаотично) и среды, в которую помещены частицы. Для усиления нелинейного сигнала часто прибегают к нанесению красителей, обладающих нелинейными оптическими свойствами, на исследуемые частицы [2-7]. Чаще других в научной литературе в роли красителей выступают малахитовый зеленый и пиридин. В работах [2-7] экспериментально исследованы их нелинейные свойства при генерации второй гармоники.

Одним из важнейших параметров, влияющих на нелинейные эффекты второго порядка от поверхности частицы, является ее размер [2-9]. Поэтому роль модели Релея-Ганса-Дебая возросла, когда стало возможным экспериментально зафиксировать излучение второй гармоники от поверхности частиц размером порядка $50 \mathrm{~nm}$ [4]. Сложности с этим возникали из-за того, что для малых частиц (значительно меньших длины волны падающего излучения) молекулы красителя, не обладающего киральными свойствами, на противоположных концах диаметра испускают электромагнитные волны, находящиеся почти в противофазе, вызывая значительное ослабление регистрируемого сигнала.
Нелинейные оптические эффекты от поверхностей могут быть использованы для определения поверхностной плотности адсорбированного вещества, поверхностного потенциала, свободной энергии [10], пространственной ориентации адсорбированных молекул [11]. Причем это возможно для адсорбированных красителей и поверхностно активных веществ на поверхности полистироловых шариков $[2,3,7]$. В медицине с помощью нелинейных оптических эффектов были исследованы мембраны липосом [12] и других везикул [13].

\section{Дипольная модель}

\section{Приближение Релея-Ганса-Дебая}

В дипольной модели генерация гармоники суммарной частоты математически описывается нелинейной частью поляризации $\mathbf{P}^{(2)}$, компоненты которой в общем виде можно записать с использованием правила суммирования по повторяющимся индексам:

$$
P_{i}^{(2)}=\chi_{i j k}^{(2)} E_{j}^{(1)} E_{k}^{(2)}
$$

где $\chi_{i j k}^{(2)}-$ тензор диэлектрической восприимчивости второго порядка, $E_{j}^{(1)}, E_{k}^{(2)}-$ компоненты векторов напряженности электрического поля падающих волн. Для центросимметричных сред все компоненты тензора $\chi_{i j k}^{(2)}$ равны нулю из-за выполнения свойств симметрии относительно поворотов и инверсии, и явление генерации гармоники суммарной частоты не наблюдается.

Точный расчет гармоники суммарной частоты требует учета рассеяния на границе раздела, что сильно усложняет задачу. Поэтому мы применим приближения 
Релея-Ганса-Дебая. Оно заключается в том, что рассеяние на границе раздела считается слабым, и нелинейная часть поляризации создается только падающими электромагнитными волнами (без учета рассеянных). Данная модель накладывает ограничения на возможные значения размера слоя и показателей преломления сред:

$$
\left|\frac{n_{p}}{n_{m}}-1\right| \ll 1, \quad 4 \pi \frac{R}{\lambda}\left|\frac{n_{p}}{n_{m}}-1\right| \ll 1 .
$$

Здесь $n_{p}$ и $n_{m}$ - показатели преломления диэлектрической частицы под нелинейным слоем и окружающей среды, $R$ - характерный размер частицы (для сферической частицы - ее радиус), $\lambda$ - длина волны падающего излучения.

Общий вид падающих электромагнитных волн в задаче выберем в форме

$$
\mathbf{E}(\mathbf{x}, t)=E_{0} \mathbf{e} \exp (i \mathbf{k x}-i \omega t),
$$

где $E_{0}$ - комплексная амплитуда волны, $\mathbf{e}-$ единичный комплексный вектор поляризации (в общем случае эллиптической поляризации), $\mathbf{k}-$ волновой вектор, $\omega-$ соответствующие циклические частоты.

\section{Уравнения Максвелла}

Рассмотрим генерацию только от тонкого нелинейного слоя на поверхности диэлектрической сферы, помещенной в диэлектрик. Тогда индукция электрического поля зависит от напряженности электрического поля в точке х следующим образом (здесь и далее используется Гауссова система единиц):

$$
\mathbf{D}(\mathbf{x}, t)=\varepsilon \mathbf{E}(\mathbf{x}, t)+4 \pi \mathbf{P}^{(2)}(\mathbf{x}, t),
$$

где вектор $\mathbf{P}^{(2)}(\mathbf{x}, t)$ отличается от нуля только в объеме нелинейного слоя на поверхности сферы, $\varepsilon$ - диэлектрическая проницаемость среды. Индукция магнитного поля зависит от напряженности магнитного поля линейно ( $\mu$ - магнитная проницаемость среды):

$$
\mathbf{B}(\mathbf{x}, t)=\mu \mathbf{H}(\mathbf{x}, t) .
$$

Тогда используя уравнения Максвелла, стандартным образом вводя потенциалы $\mathbf{A}(\mathbf{x}, t)$ и $\Phi(\mathbf{x}, t)$ и используя калибровку Лоренца, получаем для векторного потенциала $\mathbf{A}(\mathbf{x}, t)$ уравнение

$$
\frac{\varepsilon \mu}{c^{2}} \frac{\partial^{2}}{\partial t^{2}} \mathbf{A}(\mathbf{x}, t)-\nabla^{2} \mathbf{A}(\mathbf{x}, t)=\mu \frac{4 \pi}{c} \frac{\partial \mathbf{P}^{(2)}(\mathbf{x}, t)}{\partial t} .
$$

Поскольку нелинейная поляризация $\mathbf{P}^{(2)}(\mathbf{x}, t)$ обусловлена падающими монохроматическими волнами, то ее зависимость от времени имеет вид $\exp \left(-i \omega_{P} t\right)$, где $\omega_{P}-$ циклическая частота гармонических колебаний вектора $\mathbf{P}^{(2)}(\mathbf{x}, t)$ (в данной работе $\omega_{P}-$ суммарная частота). Частное решение неоднородного уравнения (6) будем искать в виде $\mathbf{A}(\mathbf{x}, t)=\mathbf{A}(\mathbf{x}) \exp \left(-i \omega_{P} t\right)$, тогда для $\mathbf{A}(\mathbf{x})$ имеем неоднородное уравнение Гельмгольца

$$
\begin{gathered}
\nabla^{2} \mathbf{A}(\mathbf{x})+\varepsilon \mu k_{P}^{2} \mathbf{A}(\mathbf{x})=4 \pi i \mu k_{P} \mathbf{P}^{(2)}(\mathbf{x}) \\
k_{P}=\omega_{P} / c
\end{gathered}
$$

из которого векторный потенциал $\mathbf{A}(\mathbf{x})$ находится методом функции Грина [14]:

$$
\mathbf{A}(\mathbf{x})=-i k_{P} \mu \int_{V} \frac{e^{i k_{P} \sqrt{\varepsilon \mu}\left|\mathbf{x}-\mathbf{x}^{\prime}\right|}}{\left|\mathbf{x}-\mathbf{x}^{\prime}\right|} \mathbf{P}^{(2)}\left(\mathbf{x}^{\prime}\right) d^{3} \mathbf{x}^{\prime} .
$$

Затем воспользуемся приближением для дальней зоны:

$$
\begin{gathered}
\exp \left(i k_{P}\left|\mathbf{x}-\mathbf{x}^{\prime}\right|\right) \approx \exp \left(i k_{P} r\right) \exp \left(-i \mathbf{k}^{(P)} \mathbf{x}^{\prime}\right), \\
|\mathbf{x}| \gg\left|\mathbf{x}^{\prime}\right|,
\end{gathered}
$$

где $r=|\mathbf{x}|$, а вектор $\mathbf{k}^{(P)}=k_{P} \sqrt{\varepsilon \mu} \mathbf{e}_{r}$ выражается через $\mathbf{e}_{r}$ - один из базисных векторов $\left(\mathbf{e}_{r}, \mathbf{e}_{\theta}, \mathbf{e}_{\varphi}\right)$ сферической системы координат $(r, \theta, \varphi)$, тогда

$$
\begin{aligned}
\mathbf{A}(\mathbf{x})= & -i k_{P} \mu \frac{e^{i k_{P} \sqrt{\varepsilon \mu} r}}{r} \\
& \times \int_{V} \exp \left(-i \mathbf{k}^{(P)} \mathbf{x}^{\prime}\right) \mathbf{P}^{(2)}\left(\mathbf{x}^{\prime}\right) d^{3} \mathbf{x}^{\prime} .
\end{aligned}
$$

Индукцию магнитного поля и напряженность электрического поля генерируемой волны в дальней зоне можно найти затем по следующим формулам:

$$
\begin{aligned}
\mathbf{B}(\mathbf{x})= & \operatorname{rot} \mathbf{A}(\mathbf{x}) \approx \mu k_{P}^{2} \sqrt{\varepsilon \mu} \frac{e^{i k_{P} \sqrt{\varepsilon \mu} r}}{r} \\
& \times\left[\mathbf{e}_{r} \times \int_{V} \exp \left(-i \mathbf{k}^{(P)} \mathbf{x}^{\prime}\right) \mathbf{P}^{(2)}\left(\mathbf{x}^{\prime}\right) d^{3} \mathbf{x}^{\prime}\right], \\
\mathbf{E}(\mathbf{x})= & \frac{i}{\left(\frac{\omega}{c} \varepsilon \mu\right)} \operatorname{rot} \mathbf{B}(\mathbf{x}) \approx \mu k_{P}^{2} \frac{e^{i k_{P} \sqrt{\varepsilon \mu} r}}{r} \\
\times & {\left.\left[\mathbf{e}_{r} \times \int_{V} \exp \left(-i \mathbf{k}^{(P)} \mathbf{x}^{\prime}\right) \mathbf{P}^{(2)}\left(\mathbf{x}^{\prime}\right) d^{3} \mathbf{x}^{\prime}\right] \times \mathbf{e}_{r}\right] } \\
= & \mu k_{P}^{2} \frac{e^{i k_{P} \sqrt{\varepsilon \mu} r}}{r}\left(1-\mathbf{e}_{r} \otimes \mathbf{e}_{r}\right) \\
& \times \int_{V} \exp \left(-i \mathbf{k}^{(P)} \mathbf{x}^{\prime}\right) \mathbf{P}^{(2)}\left(\mathbf{x}^{\prime}\right) d^{3} \mathbf{x}^{\prime},
\end{aligned}
$$

где символ $\otimes$ означает тензорное произведение векторов, а для векторного произведения используется обозначение $[\mathbf{a} \times \mathbf{b}]$, где $\mathbf{a}$ и $\mathbf{b}-$ произвольные векторы. 


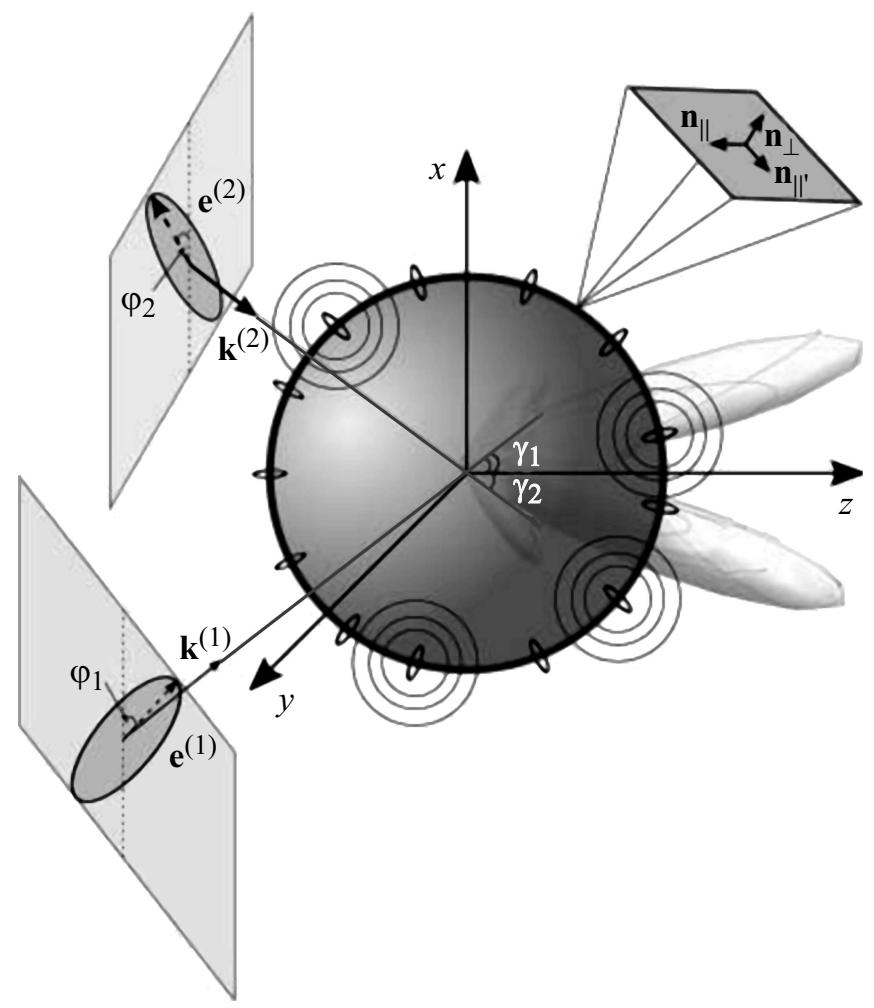

Схема задачи о генерации излучения суммарной частоты.

\section{Генерация гармоники суммарной частоты в обобщенной модели Релея-Ганса-Дебая}

\section{Аналитическое решение}

Для решения задачи используем подход, описанный нами в работах $[15,16]$. Расположим сферическую частицу радиуса $a$, покрытую тонким нелинейным слоем толщины $d_{0} \ll a$, таким образом, чтобы ее центр совпадал с центром сферической системы координат (рисунок). Записав выражение (12) для напряженности электрического поля гармоники суммарной частоты $\mathbf{E}^{(12)}(\mathbf{x})$, получим

$$
\begin{aligned}
\mathbf{E}^{(12)}(\mathbf{x})= & \left(1-\mathbf{e}_{r} \otimes \mathbf{e}_{r}\right) \mu_{12} \frac{\left(\omega_{12}\right)^{2}}{c^{2}} \frac{\exp \left(i k_{12} r\right)}{r} \\
& \times \int_{V} \exp \left(-i \mathbf{k}^{(12)} \mathbf{x}^{\prime}\right) \mathbf{P}^{(2)}\left(\mathbf{x}^{\prime}\right) d \mathbf{x}^{\prime},
\end{aligned}
$$

где $\omega_{12}=\omega_{1}+\omega_{2}-$ циклическая частота генерируемых волн, $\mathbf{k}^{(12)}$ - волновой вектор гармоники суммарной частоты, $k_{12}=\left|\mathbf{k}^{(12)}\right|=\sqrt{\varepsilon_{12} \mu_{12}} \frac{\omega_{12}}{c}, \varepsilon_{12}, \mu_{12}$ - диэлектрическая и магнитная проницаемости среды на частоте $\omega_{12}$. Интегрирование ведется по всему объему нелинейного сферического слоя $V$.
Пусть векторы напряженности электрического поля падающих электромагнитных волн задаются уравнениями:

$$
\begin{aligned}
& \mathbf{E}^{(1)}(\mathbf{x})=E_{1} \mathbf{e}^{(1)} \exp \left(i \mathbf{k}^{(1)} \mathbf{x}\right), \\
& \mathbf{E}^{(2)}(\mathbf{x})=E_{2} \mathbf{e}^{(2)} \exp \left(i \mathbf{k}^{(2)} \mathbf{x}\right),
\end{aligned}
$$

где $\mathbf{k}^{(1)}$ и $\mathbf{k}^{(2)}$ - волновые векторы падающих электромагнитных волн, а $\mathbf{e}^{(1)}$ и $\mathbf{e}^{(2)}$ - векторы поляризации падающих волн. Обозначения аналогичны указанным для уравнения (3). Тогда, подставляя (14) в (1), а результат в (13), получим

$$
\begin{aligned}
E_{i}^{(12)}(\mathbf{x})= & \mu_{12} \frac{\left(\omega_{12}\right)^{2}}{c^{2}} \frac{\exp \left(i k_{12} r\right)}{r} E_{1} E_{2} \\
& \times\left(\delta_{i m}-e_{r, i} e_{r, m}\right) e_{j}^{(1)} e_{k}^{(2)} \int_{4 \pi} d \Omega_{\mathbf{x}^{\prime}} \\
& \times \int_{a}^{a+d_{0}} \exp \left(i \mathbf{q} \mathbf{x}^{\prime}\right) \chi_{m j k}^{(2)}\left(\mathbf{x}^{\prime}\right) r^{\prime 2} d r^{\prime},
\end{aligned}
$$

где $\mathbf{q}$ - вектор рассеяния, вычисляемый по формуле

$$
\mathbf{q}=\mathbf{k}^{(1)}+\mathbf{k}^{(2)}-\mathbf{k}^{(12)}, \quad q=|\mathbf{q}| .
$$

После вычисления интеграла по $r^{\prime}$ в (15) получаем выражение, где интегрирование ведется по телесному углу:

$$
\begin{aligned}
E_{i}^{(12)}(\mathbf{x})= & \mu_{12} \frac{\left(\omega_{12}\right)^{2}}{c^{2}} \frac{\exp \left(i k_{12} r\right)}{r} d_{0} a^{2} E_{1} E_{2} \\
& \times\left(\delta_{i m}-e_{r, i} e_{r, m}\right) e_{j}^{(1)} e_{k}^{(2)} \\
& \times \int_{4 \pi} \exp \left(i \mathbf{q} \mathbf{x}^{\prime}\right) \chi_{m j k}^{(2)}\left(\mathbf{x}^{\prime}\right) d \Omega_{\mathbf{x}^{\prime}}
\end{aligned}
$$

Тензор $\chi_{m j k}^{(2)}$ в выражении (17) в самом общем виде содержит 27 компонент. Выполнение свойств симметрии при поворотах и инверсии приводят к тому, что только 7 из них остаются независимыми. Тогда тензор нелинейной диэлектрической восприимчивости второго порядка для поверхности можно представить в виде

$$
\begin{aligned}
\chi_{i j k}^{(2)}= & \chi_{1}^{(2)} n_{i} n_{j} n_{k}+\chi_{2}^{(2)} n_{i} \delta_{j k} \\
& +\chi_{3}^{(2)} n_{j} \delta_{k i}+\chi_{4}^{(2)} n_{k} \delta_{i j}+\chi_{5}^{(2)} n_{m} n_{i} \varepsilon_{m j k} \\
& +\chi_{6}^{(2)} n_{m} n_{k} \varepsilon_{i j m}+\chi_{7}^{(2)} n_{m} n_{j} \varepsilon_{i m k} .
\end{aligned}
$$

Здесь $n_{i}-$ компоненты вектора нормали $\mathbf{n}$ к поверхности, $\delta_{i j}$ - дельта-символ Кронекера, $\varepsilon_{i j k}-$ символ Леви-Чивита, $\chi_{1-7}^{(2)}-$ значения независимых 
компонент тензора диэлектрической восприимчивости. Последние три коэффициента $\chi_{5-7}^{(2)}$ в (18) называют киральными, они возможны только при наличии у слоя киральных свойств. В отсутствие этих свойств $\left(\chi_{5-7}^{(2)}=0\right)$ поверхность обладает зеркальной симметрией. Коэффициенты $\chi_{1-7}^{(2)}$ связаны с также широко используемыми в научной литературе $[4-6,8,17,18]$ компонентами тензора диэлектрической восприимчивости следующим образом (вывод формул в Приложении А):

$$
\begin{gathered}
\chi_{1}^{(2)}=\chi_{\perp \perp \perp}^{(2)}-\chi_{\perp\|\|}^{(2)}-\chi_{\|\perp\|}^{(2)}-\chi_{\|\| \perp}^{(2)}, \\
\chi_{2}^{(2)}=\chi_{\perp\|\|}^{(2)}=\chi_{\perp\left\|^{\prime}\right\|^{\prime}}^{(2)}, \quad \chi_{3}^{(2)}=\chi_{\|\perp\|}^{(2)}=\chi_{\left\|^{\prime} \perp\right\|^{\prime}}^{(2)}, \\
\chi_{4}^{(2)}=\chi_{\|\| \perp}^{(2)}=\chi_{\left\|^{\prime}\right\|^{\prime} \perp}^{(2)}, \\
\chi_{5}^{(2)}=\chi_{\perp\|\|^{\prime}}^{(2)}=-\chi_{\perp\left\|^{\prime}\right\|}^{(2)}, \\
\chi_{6}^{(6)}=\chi_{\|\|^{\prime} \perp}^{(2)}=-\chi_{\left\|^{\prime}\right\| \perp}^{(2)}, \\
\chi_{7}^{(2)}=\chi_{\left\|^{\prime} \perp\right\|}^{(2)}=-\chi_{\|\perp\|^{\prime}}^{(2)},
\end{gathered}
$$

где $\perp,\|,\|^{\prime}-$ компоненты тензора вдоль осей, сонаправленных базисным векторам $\mathbf{n}_{\perp}=\mathbf{n}, \mathbf{n}_{\|}, \mathbf{n}_{\|^{\prime}}$, где $\mathbf{n}_{\perp}$ перпендикулярен поверхности, а $\mathbf{n}_{\|}, \mathbf{n}_{\|^{\prime}}$ параллельны поверхности, причем $\mathbf{n}_{\perp}, \mathbf{n}_{\|}, \mathbf{n}_{\|^{\prime}}$ образуют правую тройку векторов (рисунок). В дальнейшем для расчетов будут использоваться коэффициенты $\chi_{1-7}^{(2)}$, так как в этом случае описывающие генерацию формулы имеют более простой вид.

После подстановки (18) в (17) получаем выражение с тремя следующими интегралами (подробный вывод в Приложении В), где индексы $i, j, k=x, y, z$ :

$$
\begin{gathered}
\int_{4 \pi} \exp (i a \mathbf{q n}) n_{k} d \Omega_{\mathbf{x}^{\prime}}=4 \pi i v_{k} j_{1}(q a) \\
\int_{4 \pi} \exp (i a \mathbf{q n}) n_{i} n_{j} d \Omega_{\mathbf{x}^{\prime}} \\
=4 \pi\left[\delta_{i j} \frac{1}{3}\left(j_{0}(q a)+j_{2}(q a)\right)-v_{i} v_{j} j_{2}(q a)\right], \\
\int_{4 \pi} \exp (i a \mathbf{q n}) n_{i} n_{j} n_{k} d \Omega_{\mathbf{x}^{\prime}}=4 \pi i\left[\frac{1}{5}\left(j_{1}(q a)+j_{3}(q a)\right)\right. \\
\left.\times\left(v_{i} \delta_{j k}+v_{j} \delta_{k i}+v_{k} \delta_{i j}\right)-j_{3}(q a) v_{i} v_{j} v_{k}\right]
\end{gathered}
$$

где $j_{m}(z)$ - сферические функции Бесселя порядка $m, v_{i}$ - компоненты единичного вектора вдоль q, а символ $i$ вне индексов означает мнимую единицу. Пользуясь (20)-(22), получаем выражение для тензора эффективной нелинейной диэлектрической восприимчивости:

$$
\begin{aligned}
\mathrm{X}_{i j k}^{(12)} & =\frac{1}{4 \pi} \int_{4 \pi} \exp \left(i \mathbf{q} \mathbf{x}^{\prime}\right) \chi_{i j k}^{(2)}\left(\mathbf{x}^{\prime}\right) d \Omega_{\mathbf{x}^{\prime}} \\
& =i \chi_{1}^{(2)}\left[-j_{3}(q a) v_{i} v_{j} v_{k}+\frac{1}{5}\left(j_{1}(q a)+j_{3}(q a)\right)\right. \\
& \left.\times\left(v_{i} \delta_{j k}+v_{j} \delta_{k i}+v_{k} \delta_{i j}\right)\right] \\
& +i j_{1}(q a)\left[\chi_{2}^{(2)} v_{i} \delta_{j k}+\chi_{3}^{(2)} v_{j} \delta_{i k}+\chi_{4}^{(2)} v_{k} \delta_{i j}\right] \\
& +\frac{1}{3}\left(j_{0}(q a)+j_{2}(q a)\right)\left(\chi_{5}^{(2)}+\chi_{6}^{(2)}+\chi_{7}^{(2)}\right) \varepsilon_{i j k} \\
& -j_{2}(q a)\left(\chi_{5}^{(2)} v_{i} v_{m} \varepsilon_{m j k}+\chi_{6}^{(2)} v_{k} v_{m} \varepsilon_{i j m}\right. \\
& \left.+\chi_{7}^{(2)} v_{j} v_{m} \varepsilon_{i m k}\right) .
\end{aligned}
$$

Вектор $\mathbf{f}^{(12)}$, компоненты которого определяются по формуле $f_{i}^{(12)}=\mathrm{X}_{i j k}^{(12)} e_{j}^{(1)} e_{k}^{(2)}$, запишется следующим образом:

$$
\begin{aligned}
\mathbf{f}^{(12)}= & i \chi_{1}^{(2)}\left(-j_{3}(q a) \boldsymbol{v}\left(\boldsymbol{v} \mathbf{e}^{(1)}\right)\left(\boldsymbol{v} \mathbf{e}^{(2)}\right)\right. \\
& +\frac{1}{5}\left(j_{1}(q a)+j_{3}(q a)\right) \\
& \left.\times\left(\boldsymbol{v}\left(\mathbf{e}^{(1)} \mathbf{e}^{(2)}\right)+\mathbf{e}^{(2)}\left(\boldsymbol{v} \mathbf{e}^{(1)}\right)+\mathbf{e}^{(1)}\left(\boldsymbol{v} \mathbf{e}^{(2)}\right)\right)\right) \\
& +i j_{1}(q a)\left(\chi_{2}^{(2)} \boldsymbol{v}\left(\mathbf{e}^{(1)} \mathbf{e}^{(2)}\right)+\chi_{3}^{(2)} \mathbf{e}^{(2)}\left(\boldsymbol{v} \mathbf{e}^{(1)}\right)\right. \\
& \left.+\chi_{4}^{(2)} \mathbf{e}^{(1)}\left(\boldsymbol{v} \mathbf{e}^{(2)}\right)\right)+\frac{1}{3}\left(j_{0}(q a)+j_{2}(q a)\right) \\
& \times\left(\chi_{5}^{(2)}+\chi_{6}^{(2)}+\chi_{7}^{(2)}\right)\left[\mathbf{e}^{(1)} \times \mathbf{e}^{(2)}\right] \\
& -j_{2}(q a)\left(\chi_{5}^{(2)} \boldsymbol{v}\left(\boldsymbol{v}\left[\mathbf{e}^{(1)} \times \mathbf{e}^{(2)}\right]\right)\right. \\
& \left.+\chi_{7}^{(2)}\left[\boldsymbol{v} \times \mathbf{e}^{(2)}\right]\left(\boldsymbol{v} \mathbf{e}^{(1)}\right)\right) . \\
& +\chi_{6}^{(2)}\left[\mathbf{e}^{(1)} \times \boldsymbol{v}\right]\left(\boldsymbol{v} \mathbf{e}^{(2)}\right) \\
& \\
&
\end{aligned}
$$

Можно заметить, что киральная часть вектора $\mathbf{f}^{(12)}$ в (24) отличается от некиральной по фазе на множитель $i$, как и при генерации второй гармоники. 
Тогда формула для вектора электрического поля гармоники суммарной частоты принимает следующий вид:

$$
\begin{aligned}
\mathbf{E}^{(12)}(\mathbf{x})= & 4 \pi \mu_{12} \frac{\omega_{12}^{2}}{c^{2}} \frac{\exp \left(i k_{12} r\right)}{r} \\
& \times d_{0} a^{2} E_{1} E_{2}\left(1-\mathbf{e}_{r} \otimes \mathbf{e}_{r}\right) \mathbf{f}^{(12)}
\end{aligned}
$$

Магнитную индукцию $\mathbf{B}^{(12)}$ поля гармоники суммарной частоты, а затем магнитную напряженность $\mathbf{H}^{(12)}$ можно найти по формуле (11), проделывая совершенно аналогичные вычисления. Но можно также найти вектор магнитной напряженности $\mathbf{H}^{(12)}$, используя (25) и уравнения Максвелла. В обоих случаях получаем одинаковый результат:

$$
\begin{aligned}
\mathbf{H}^{(12)}(\mathbf{x}) & =\frac{1}{\mu_{12}} \frac{c}{i \omega_{12}} \operatorname{rot} \mathbf{E}^{(12)}(\mathbf{x}) \\
& =\frac{n_{12}}{\mu_{12}}\left[\mathbf{e}_{r} \times \mathbf{E}^{(12)}(\mathbf{x})\right],
\end{aligned}
$$

где $n_{12}$ - показатель преломления окружающей частицу среды на частоте $\omega_{12}$.

Наиболее удобной для наблюдения характеристикой излучения является его мощность, поэтому целесообразно получить выражение для радиальной компоненты вектора Умова-Пойнтинга в дальней зоне на основе выражений (25) и (26):

$$
\begin{aligned}
S_{r}^{(12)}(\mathbf{x}) & =\frac{1}{2} \frac{c}{4 \pi} \operatorname{Re}\left[\mathbf{E}^{(12)}(\mathbf{x}) \times\left(\mathbf{H}^{(12)}(\mathbf{x})\right)^{*}\right]_{r} \\
& =\frac{c}{8 \pi} \frac{n_{12}}{\mu_{12}}\left|\mathbf{E}^{(12)}(\mathbf{x})\right|^{2} .
\end{aligned}
$$

Здесь символ $*$ означает комплексное сопряжение.

В частном случае, когда $\gamma=0, \omega_{1}=\omega_{2}, \mathbf{k}^{(1)}=\mathbf{k}^{(2)}$, $\varphi_{\text {in }}^{(1)}=\varphi_{\text {in }}^{(2)}, \sigma_{1}=\sigma_{2}, E_{1}=E_{2}$ описанное решение задачи о генерации суммарной частоты совпадают с решением задачи о генерации второй гармоники [16].

\section{Предельные формы решения}

Для анализа поведения вектора $\mathbf{f}^{(12)}$ при предельных значениях радиуса частицы $a$ будем пользоваться приближенными выражениями сферических функций Бесселя для малых и больших значений аргумента $z$ :

$$
\begin{gathered}
j_{m}(z)=\frac{z^{m}}{(2 n+1) ! !}, \quad z \ll 1, \\
j_{m}(z)=\frac{\sin (z-\pi m / 2)}{z}, \quad z \gg 1 .
\end{gathered}
$$

Тогда вид вектора $\mathbf{f}^{(12)}$ при малых значениях радиуса сферического слоя $(q a \ll 1)$

$$
\begin{aligned}
& \mathbf{f}^{(12)}=i \frac{q a}{15} \chi_{1}^{(2)}\left(\boldsymbol{v}\left(\mathbf{e}^{(1)} \mathbf{e}^{(2)}\right)+\mathbf{e}^{(2)}\left(\boldsymbol{v} \mathbf{e}^{(1)}\right)+\mathbf{e}^{(1)}\left(\boldsymbol{v} \mathbf{e}^{(2)}\right)\right) \\
& +i \frac{q a}{3}\left(\chi_{2}^{(2)} \boldsymbol{v}\left(\mathbf{e}^{(1)} \mathbf{e}^{(2)}\right)+\chi_{3}^{(2)} \mathbf{e}^{(2)}\left(\boldsymbol{v} \mathbf{e}^{(1)}\right)+\chi_{4}^{(2)} \mathbf{e}^{(1)}\left(\boldsymbol{v} \mathbf{e}^{(2)}\right)\right) \\
& +\frac{1}{3}\left(\chi_{5}^{(2)}+\chi_{6}^{(2)}+\chi_{7}^{(2)}\right)\left[\mathbf{e}^{(1)} \times \mathbf{e}^{(2)}\right],
\end{aligned}
$$

а при больших значениях радиуса сферического слоя $(q a \gg 1)$

$$
\begin{aligned}
\mathbf{f}^{(12)} & =-\frac{\cos (q a)}{q a} i\left[\chi_{1}^{(2)} \boldsymbol{v}\left(\boldsymbol{v} \mathbf{e}^{(1)}\right)\left(\boldsymbol{v} \mathbf{e}^{(2)}\right)+\chi_{2}^{(2)} v\left(\mathbf{e}^{(1)} \mathbf{e}^{(2)}\right)\right. \\
& \left.+\chi_{3}^{(2)} \mathbf{e}^{(2)}\left(\boldsymbol{v} \mathbf{e}^{(1)}\right)+\chi_{4}^{(2)} \mathbf{e}^{(1)}\left(\boldsymbol{v} \mathbf{e}^{(2)}\right)\right] \\
& +\frac{\sin (q a)}{q a}\left(\chi_{5}^{(2)} \boldsymbol{v}\left(\boldsymbol{v}\left[\mathbf{e}^{(1)} \times \mathbf{e}^{(2)}\right]\right)\right. \\
& \left.+\chi_{6}^{(2)}\left[\mathbf{e}^{(1)} \times \boldsymbol{v}\right]\left(\boldsymbol{v} \mathbf{e}^{(2)}\right)+\chi_{7}^{(2)}\left[\boldsymbol{v} \times \mathbf{e}^{(2)}\right]\left(\boldsymbol{v} \mathbf{e}^{(1)}\right)\right) .
\end{aligned}
$$

Согласно формуле (29), при малых размерах частицы доминирующую роль в генерации гармоники суммарной частоты будут иметь киральные компоненты. Из формулы (27) также получаем, что $S_{r}^{(12)} \sim a^{4}$. Если же нелинейный слой не обладает киральными свойствами, то $S_{r}^{(12)} \sim a^{6}$. Также можно заметить, что излучение, обусловленное некиральными компонентами, отсутствует в направлении, для которого $q=0$, тогда как для киральных компонент излучение в этом направлении сохраняется. Выражение (30) дает только приблизительную оценку того, как будет меняться диаграмма направленности (характеризует пространственное распределение мощности генерируемого излучения) при увеличении размеров частицы, так как приближение Релея-Ганса-Дебая применимо для больших по сравнению с длиной волны частиц только при хорошем совпадении показателей преломления внутри и вне частицы. Функции $\cos (q a) /(q a)$ и $\sin (q a) /(q a)$ имеют ряд максимумов убывающей амплитуды при росте $q$, т.е. при отклонении от направления $q=0$. На диаграмме направленности это проявится как множество побочных лепестков. При этом лепесток максимальной длины на диаграмме направленности будет приближаться к направлению $q=0$ и становиться уже с увеличением радиуса частицы $a$. Для слоя, обладающего только киральными свойствами $\left(\chi_{1-4}^{(2)}=0\right)$, этот лепесток, обусловленный слагаемым $\frac{1}{3}\left(\chi_{5}^{(2)}+\chi_{6}^{(2)}+\chi_{7}^{(2)}\right)\left[\mathbf{e}^{(1)} \times \mathbf{e}^{(2)}\right]$ в (29), будет расположен в направлении $q=0$ и также становится уже с увеличением радиуса частицы $a$. 


\section{Заключение}

В настоящей работе с использованием приближения Релея-Ганса-Дебая получены явные выражения для поля суммарной частоты в дальней зоне. Эти выражения можно будет использовать для вычисления вклада каждой из сферических поверхностей, входящих в состав исследуемой частицы, например многослойной частицы. Учет дисперсии, произведенный в полученном решении, расширяет пределы применимости данной модели, что для генерации второй гармоники было показано ранее [6]. Все аналитические формулы, полученные в работе, проверены численно.

Анализ предельных форм решения для больших и малых радиусов сферического слоя показал, что при генерации суммарной частоты от сферического слоя малого радиуса именно излучение, обусловленное киральными коэффициентами $\chi_{5-7}^{(2)}$, вносит наибольший вклад в генерируемое поле. Также обнаружено, что количество максимумов в функции, характеризующей пространственное распределение мощности генерируемого излучения, растет с увеличением радиуса сферического слоя. Причем в направлении, для которого вектор рассеяния равен нулю, наблюдается минимум излучения, обусловленного некиральными коэффициентами анизотропии $\chi_{1-4}^{(2)}$, и максимум излучения, обусловленного киральными коэффициентами анизотропии $\chi_{5-7}^{(2)}$.

\section{Приложение A}

Произведение $\chi_{i j k}^{(2)} e_{j}^{(1)} e_{k}^{(2)}$, где $\mathbf{e}^{(1)}, \mathbf{e}^{(2)}-$ произвольные векторы, можно представить в векторном виде следующим образом:

$$
\begin{aligned}
& \chi_{i j k}^{(2)} e_{j}^{(1)} e_{k}^{(2)}=\chi_{1}^{(2)} n_{i}\left(n_{j} e_{j}^{(1)}\right)\left(n_{k} e_{k}^{(2)}\right) \\
& +\chi_{2}^{(2)} n_{i}\left(\delta_{j k} e_{j}^{(1)} e_{k}^{(2)}\right) \\
& +\chi_{3}^{(2)} \delta_{k i}\left(n_{j} e_{j}^{(1)}\right) e_{k}^{(2)}+\chi_{4}^{(2)} \delta_{i j} e_{j}^{(1)}\left(n_{k} e_{k}^{(2)}\right) \\
& +\chi_{5}^{(2)} n_{i} n_{m}\left(\varepsilon_{m j k} e_{j}^{(1)} e_{k}^{(2)}\right)+\chi_{6}^{(2)}\left(n_{k} e_{k}^{(2)}\right)\left(\varepsilon_{i j m} n_{m} e_{j}^{(1)}\right) \\
& +\chi_{7}^{(2)}\left(n_{j} e_{j}^{(1)}\right)\left(n_{m} \varepsilon_{i m k} e_{k}^{(2)}\right)=\left[\chi_{1}^{(2)} \mathbf{n}\left(\mathbf{n} \mathbf{e}^{(1)}\right)\left(\mathbf{n} \mathbf{e}^{(2)}\right)\right. \\
& +\chi_{2}^{(2)} \mathbf{n}\left(\mathbf{e}^{(1)} \mathbf{e}^{(2)}\right)+\chi_{3}^{(2)} \mathbf{e}^{(2)}\left(\mathbf{n} \mathbf{e}^{(1)}\right)+\chi_{4}^{(2)} \mathbf{e}^{(1)}\left(\mathbf{n} \mathbf{e}^{(2)}\right) \\
& +\chi_{5}^{(2)} \mathbf{n}\left(\mathbf{n}\left[\mathbf{e}^{(1)} \times \mathbf{e}^{(2)}\right]\right)+\chi_{6}^{(2)}\left[\mathbf{e}^{(1)} \times \mathbf{n}\right]\left(\mathbf{n} \mathbf{e}^{(2)}\right) \\
& \left.+\chi_{7}^{(2)}\left[\mathbf{n} \times \mathbf{e}^{(2)}\right]\left(\mathbf{n} \mathbf{e}^{(1)}\right)\right]_{i}
\end{aligned}
$$

Запишем полученное в (31) выражение через компоненты вдоль осей декартовой системы координат, заданной векторами $\mathbf{n}_{\perp}, \mathbf{n}_{\|}, \mathbf{n}_{\| \prime}$ :

$$
\begin{aligned}
& \chi_{1}^{(2)} \mathbf{n}\left(\mathbf{n} \mathbf{e}^{(1)}\right)\left(\mathbf{n} \mathbf{e}^{(2)}\right)+\chi_{2}^{(2)} \mathbf{n}\left(\mathbf{e}^{(1)} \mathbf{e}^{(2)}\right)+\chi_{3}^{(2)} \mathbf{e}^{(2)}\left(\mathbf{n} \mathbf{e}^{(1)}\right) \\
& +\chi_{4}^{(2)} \mathbf{e}^{(1)}\left(\mathbf{n} \mathbf{e}^{(2)}\right)+\chi_{5}^{(2)} \mathbf{n}\left(\mathbf{n}\left[\mathbf{e}^{(1)} \times \mathbf{e}^{(2)}\right]\right) \\
& +\chi_{6}^{(2)}\left[\mathbf{e}^{(1)} \times \mathbf{n}\right]\left(\mathbf{n} \mathbf{e}^{(2)}\right)+\chi_{7}^{(2)}\left[\mathbf{n} \times \mathbf{e}^{(2)}\right]\left(\mathbf{n} \mathbf{e}^{(1)}\right) \\
& =\chi_{1}^{(2)} \mathbf{n}_{\perp} e_{\perp}^{(1)} e_{\perp}^{(2)}+\chi_{2}^{(2)} \mathbf{n}_{\perp}\left(e_{\perp}^{(1)} e_{\perp}^{(2)}+e_{\|}^{(1)} e_{\|}^{(2)}+e_{\|^{\prime}}^{(1)} e_{\|^{\prime}}^{(2)}\right) \\
& +\chi_{3}^{(2)}\left(\mathbf{n}_{\perp} e_{\perp}^{(2)}+\mathbf{n}_{\|} e_{\|}^{(2)}+\mathbf{n}_{\|^{\prime}} e_{\|^{\prime}}^{(2)}\right) e_{\perp}^{(1)} \\
& +\chi_{4}^{(2)}\left(\mathbf{n}_{\perp} e_{\perp}^{(1)}+\mathbf{n}_{\|} e_{\|}^{(1)}+\mathbf{n}_{\|^{\prime}} e_{\|^{\prime}}^{(1)}\right) e_{\perp}^{(2)} \\
& +\chi_{5}^{(2)} \mathbf{n}_{\perp}\left(e_{\|}^{(1)} e_{\|^{\prime}}^{(2)}-e_{\|^{\prime}}^{(1)} e_{\|}^{(2)}\right)+\chi_{6}^{(2)}\left(\mathbf{n}_{\|} e_{\|^{\prime}}^{(1)}-\mathbf{n}_{\|^{\prime}} e_{\|}^{(1)}\right) e_{\perp}^{(2)} \\
& +\chi_{7}^{(2)} e_{\perp}^{(1)}\left(\mathbf{n}_{\|^{\prime}} e_{\|}^{(2)}-\mathbf{n}_{\|} e_{\|^{\prime}}^{(2)}\right) \\
& =\mathbf{n}_{\perp}\left(\chi_{1}^{(2)}+\chi_{2}^{(2)}+\chi_{3}^{(2)}+\chi_{4}^{(2)}\right) e_{\perp}^{(1)} e_{\perp}^{(2)} \\
& +\mathbf{n}_{\perp} \chi_{2}^{(2)}\left(e_{\|}^{(1)} e_{\|}^{(2)}+e_{\|^{\prime}}^{(1)} e_{\|^{\prime}}^{(2)}\right)+\chi_{3}^{(2)} e_{\perp}^{(1)}\left(\mathbf{n}_{\|} e_{\|}^{(2)}+\mathbf{n}_{\|^{\prime}} e_{\|^{\prime}}^{(2)}\right) \\
& +\chi_{4}^{(2)}\left(\mathbf{n}_{\|} e_{\|}^{(1)}+\mathbf{n}_{\|^{\prime}} e_{\|^{\prime}}^{(1)}\right) e_{\perp}^{(2)}+\chi_{5}^{(2)} \mathbf{n}_{\perp}\left(e_{\|}^{(1)} e_{\|^{\prime}}^{(2)}-e_{\|^{\prime}}^{(1)} e_{\|}^{(2)}\right) \\
& +\chi_{6}^{(2)}\left(\mathbf{n}_{\|} e_{\|^{\prime}}^{(1)}-\mathbf{n}_{\|^{\prime}} e_{\|}^{(1)}\right) e_{\perp}^{(2)}+\chi_{7}^{(2)} e_{\perp}^{(1)}\left(\mathbf{n}_{\|^{\prime}} e_{\|}^{(2)}-\mathbf{n}_{\|} e_{\|^{\prime}}^{(2)}\right) \\
& =\mathbf{n}_{\perp} \chi_{\perp \perp \perp}^{(2)} e_{\perp}^{(1)} e_{\perp}^{(2)}+\mathbf{n}_{\perp} \chi_{\perp\|\|}^{(2)} e_{\|}^{(1)} e_{\|}^{(2)}+\mathbf{n}_{\perp} \chi_{\perp\left\|^{\prime}\right\|^{\prime}}^{(2)} e_{\|^{\prime}}^{(1)} e_{\|^{\prime}}^{(2)} \\
& +\mathbf{n}_{\|} \chi_{\|\perp\|}^{(2)} e_{\perp}^{(1)} e_{\|}^{(2)}+\mathbf{n}_{\|^{\prime}} \chi_{\left\|^{\prime} \perp\right\|^{\prime}}^{(2)} e_{\perp}^{(1)} e_{\|^{\prime}}^{(2)}+\mathbf{n}_{\|} \chi_{\|\| \perp}^{(2)} e_{\|}^{(1)} e_{\perp}^{(2)} \\
& +\mathbf{n}_{\|^{\prime}} \chi_{\left\|^{\prime}\right\|^{\prime} \perp}^{(2)} e_{\|^{\prime}}^{(1)} e_{\perp}^{(2)}+\mathbf{n}_{\perp} \chi_{\perp\|\| \|^{\prime}}^{(2)} e_{\|}^{(1)} e_{\|^{\prime}}^{(2)}-\mathbf{n}_{\perp} \chi_{\perp\left\|^{\prime}\right\|}^{(2)} e_{\|^{\prime}}^{(1)} e_{\|}^{(2)} \\
& +\mathbf{n}_{\|} \chi_{\|\|^{\prime} \perp}^{(2)} e_{\|^{\prime}}^{(1)} e_{\perp}^{(2)}-\mathbf{n}_{\| \prime} \chi_{\|\|^{\prime} \| \perp}^{(2)} e_{\|}^{(1)} e_{\perp}^{(2)} \\
& +\mathbf{n}_{\|} \chi_{\left\|^{\prime} \perp\right\|}^{(2)} e_{\perp}^{(1)} e_{\|}^{(2)}-\mathbf{n}_{\|} \chi_{\|\perp\|^{\prime}}^{(2)} e_{\perp}^{(1)} e_{\|^{\prime}}^{(2)} \text {. }
\end{aligned}
$$

Здесь выполнена следующая замена:

$$
\begin{gathered}
\chi_{\perp \perp \perp}^{(2)}=\chi_{1}^{(2)}+\chi_{2}^{(2)}+\chi_{3}^{(3)}+\chi_{4}^{(2)}, \quad \chi_{\perp\|\|}^{(2)}=\chi_{\perp\left\|^{\prime}\right\|^{\prime}}^{(2)}=\chi_{2}^{(2)}, \\
\chi_{\|\perp\|}^{(2)}=\chi_{\|\|^{\prime} \perp \|^{\prime}}^{(2)}=\chi_{3}^{(2)}, \quad \chi_{\|\| \perp}^{(2)}=\chi_{\left\|^{\prime}\right\|^{\prime} \perp}^{(2)}=\chi_{4}^{(2)}, \\
\chi_{\perp\|\|^{\prime}}^{(2)}=-\chi_{\perp\left\|^{\prime}\right\|}^{(2)}=\chi_{5}^{(2)}, \quad \chi_{\|\|^{\prime} \perp}^{(2)}=-\chi_{\left\|^{\prime}\right\| \perp}^{(2)}=\chi_{6}^{(2)}, \\
\chi_{\|\|^{\prime} \perp \|}^{(2)}=-\chi_{\|\perp\|^{\prime}}^{(2)}=\chi_{7}^{(2)} .
\end{gathered}
$$

Преобразуя формулы (33), получаем выражения (19).

\section{Приложение В}

Перейдем в систему отсчета, в которой вектор q направлен вдоль оси $O z^{\prime}$. Выражение старых базисных векторов $\left(\mathbf{e}_{x}, \mathbf{e}_{y}, \mathbf{e}_{z}\right)$ через новые $\left(\mathbf{e}_{x}^{\prime}, \mathbf{e}_{y}^{\prime}, \mathbf{e}_{z}^{\prime}\right)$ при таком 
переходе выглядит следующим образом:

$$
\left[\begin{array}{c}
\mathbf{e}_{x} \\
\mathbf{e}_{y} \\
\mathbf{e}_{z}
\end{array}\right]=\left[\begin{array}{ccc}
\cos \theta_{q} \cos \varphi_{q} & -\sin \varphi_{q} & \sin \theta_{q} \cos \varphi_{q} \\
\cos \theta_{q} \sin \varphi_{q} & \cos \varphi_{q} & \sin \theta_{q} \sin \varphi_{q} \\
-\sin \theta_{q} & 0 & \cos \theta_{q}
\end{array}\right]\left[\begin{array}{c}
\mathbf{e}_{x}^{\prime} \\
\mathbf{e}_{y}^{\prime} \\
\mathbf{e}_{z}^{\prime}
\end{array}\right] .
$$

Здесь углы $\theta_{q}, \varphi_{q}$ - угловые координаты вектора $\mathbf{q}$ в старой сферической системе координат. В новой системе координат вектор $\mathbf{n}$ запишется в виде

$$
\mathbf{n}=\sin \theta \cos \varphi \mathbf{e}_{x}^{\prime}+\sin \theta \sin \varphi \mathbf{e}_{y}^{\prime}+\cos \theta \mathbf{e}_{z}^{\prime} .
$$

Тогда его проекции на старые базисные векторы равны

$$
\begin{aligned}
n_{x}=\mathbf{n} \mathbf{e}_{x}= & \cos \theta_{q} \cos \varphi_{q} \sin \theta \cos \varphi-\sin \varphi_{q} \sin \theta \sin \varphi \\
& +\sin \theta_{q} \cos \varphi_{q} \cos \theta, \\
n_{y}=\mathbf{n e}_{y}= & \cos \theta_{q} \sin \varphi_{q} \sin \theta \cos \varphi+\cos \varphi_{q} \sin \theta \sin \varphi \\
& +\sin \theta_{q} \sin \varphi_{q} \cos \theta, \\
n_{z}=\mathbf{n e}_{z}= & -\sin \theta_{q} \sin \theta \cos \varphi+\cos \theta_{q} \cos \theta .
\end{aligned}
$$

Для вычисления (20)-(22) понадобились интегралы

$$
\begin{aligned}
& \int_{0}^{\pi} \exp (i z \cos \theta) \cos ^{m} \theta \sin \theta d \theta \\
& \quad=\frac{2}{i^{m}} \frac{d^{m}}{d z^{m}} j_{0}(z)=\frac{2}{i^{m}} j_{0}^{(m)}(z),
\end{aligned}
$$

где верхний индекс $(m)$ означает производную порядка $m$ по аргументу.

Подставляя (36) в формулу (20) для индекса $k=z$, получаем интеграл

$$
\begin{aligned}
\int_{4 \pi} & \exp (i \mathbf{q} \mathbf{x}) n_{z} d \Omega_{\mathbf{x}^{\prime}} \\
& =\int_{0}^{\pi} \sin \theta d \theta \int_{0}^{2 \pi} \exp (i q a \cos \theta)\left(\mathbf{n} \mathbf{e}_{z}\right) d \varphi \\
= & \int_{0}^{\pi} \sin \theta d \theta \int_{0}^{2 \pi} \exp (i q a \cos \theta) \\
\times & \left(-\sin \theta_{q} \sin \theta \cos \varphi+\cos \theta_{q} \cos \theta\right) d \varphi \\
= & 2 \pi \cos \theta_{q} \int_{0}^{\pi} \exp (i q a \cos \theta) \cos \theta \sin \theta d \theta \\
= & -4 \pi i \cos \theta_{q} j_{0}^{(1)}(q a)=-4 \pi i v_{z} j_{0}^{(1)}(q a) .
\end{aligned}
$$

Здесь $v_{z}-z$-компонента единичного вектора $\mathbf{v}$ вдоль вектора q в старой системе координат. Аналогично вычисляя интегралы для индексов $k=x$ и $k=y$ и применяя рекуррентное соотношение

$$
(2 m+1) j_{m}^{(1)}(z)=m j_{m-1}(z)-(m+1) j_{m+1}(z),
$$

получаем формулу (20).

Для вычисления интеграла (21) воспользуемся этим же переходом в новую систему отсчета. Рассмотрим случай $i=j=z$ :

$$
\int_{4 \pi} \exp (i \mathbf{q} \mathbf{x})\left(n_{z}\right)^{2} d \Omega_{\mathbf{x}^{\prime}}=\int_{0}^{\pi} \sin \theta d \theta \int_{0}^{2 \pi} \exp (i \mathbf{q n} a)\left(\mathbf{n e}_{z}\right)^{2} d \varphi
$$$$
=\int_{0}^{\pi} \sin \theta d \theta \int_{0}^{2 \pi} \exp (\text { iqa } \cos \theta)\left(-\sin \theta_{q} \sin \theta \cos \varphi\right.
$$$$
\left.+\cos \theta_{q} \cos \theta\right)^{2} d \varphi=\int_{0}^{\pi} \exp (\text { iqa } \cos \theta)\left(\pi \sin ^{2} \theta_{q} \sin ^{2} \theta\right.
$$$$
\left.+2 \pi \cos ^{2} \theta_{q} \cos ^{2} \theta\right) \sin \theta d \theta=\pi \sin ^{2} \theta_{q} 2\left(j_{0}(q a)+j_{0}^{(2)}(q a)\right)
$$$$
+2 \pi \cos ^{2} \theta_{q}(-2) j_{0}^{(2)}(q a)=2 \pi\left(1-v_{z}^{2}\right)
$$$$
\times\left(j_{0}(q a)+j_{0}^{(2)}(q a)\right)-4 \pi v_{z}^{2} j_{0}^{(2)}(q a) .
$$

А для $i=x, j=z$ интеграл примет вид

$$
\int_{4 \pi} \exp (i \mathbf{q} \mathbf{x}) n_{x} n_{z} d \Omega_{\mathbf{x}^{\prime}}=\int_{0}^{\pi} \sin \theta d \theta \int_{0}^{2 \pi} \exp (i \mathbf{q n} a)\left(\mathbf{n e}_{x}\right)\left(\mathbf{n e}_{z}\right) d \varphi
$$$$
=\int_{0}^{\pi} \sin \theta d \theta \int_{0}^{2 \pi} \exp (i q a \cos \theta)\left(\cos \theta_{q} \cos \varphi_{q} \sin \theta \cos \varphi\right.
$$$$
\left.-\sin \varphi_{q} \sin \theta \sin \varphi+\sin \theta_{q} \cos \varphi_{q} \cos \theta\right)
$$$$
\times\left(-\sin \theta_{q} \sin \theta \cos \varphi+\cos \theta_{q} \cos \theta\right) d \varphi
$$$$
=\int_{0}^{\pi} \sin \theta d \theta \int_{0}^{2 \pi} \exp (i q a \cos \theta)
$$$$
\times\left(-\cos \theta_{q} \sin \theta_{q} \cos \varphi_{q} \sin ^{2} \theta \cos ^{2} \varphi\right.
$$$$
\left.+\sin \theta_{q} \cos \theta_{q} \cos \varphi_{q} \cos ^{2} \theta\right) d \varphi
$$$$
=\pi\left(-\cos \theta_{q} \sin \theta_{q} \cos \varphi_{q}\right) 2\left(j_{0}(q a)+j_{0}^{(2)}(q a)\right)
$$$$
+2 \pi \sin \theta_{q} \cos \theta_{q} \cos \varphi_{q}(-2) j_{0}^{(2)}(q a)
$$$$
=-2 \pi v_{x} v_{z}\left(j_{0}(q a)+j_{0}^{(2)}(q a)\right)-4 \pi v_{x} v_{z} j_{0}^{(2)}(q a) \text {. }
$$ 
Аналогично рассматривая другие комбинации $i$ и $j$, приходим к обобщенной формуле (21).

Для нахождения интеграла (22) рассмотрим случай $i=x, j=y, k=z$ :

$$
\begin{aligned}
& \int_{4 \pi} \exp (i \mathbf{q} \mathbf{x}) n_{x} n_{y} n_{z} d \Omega_{\mathbf{x}^{\prime}}=\int_{0}^{\pi} \sin \theta d \theta \int_{0}^{2 \pi} \\
& \times \exp (i \mathbf{q n} a)\left(\mathbf{n e}_{x}\right)\left(\mathbf{n e}_{y}\right)\left(\mathbf{n e}_{z}\right) d \varphi \\
& =\int_{0}^{\pi} \sin \theta d \theta \int_{0}^{2 \pi} \exp (i q a \cos \theta)\left(\cos \theta_{q} \cos \varphi_{q} \sin \theta \cos \varphi\right. \\
& \left.-\sin \varphi_{q} \sin \theta \sin \varphi+\sin \theta_{q} \cos \varphi_{q} \cos \theta\right) \\
& \times\left(\cos \theta_{q} \sin \varphi_{q} \sin \theta \cos \varphi+\cos \varphi_{q} \sin \theta \sin \varphi\right. \\
& \left.+\sin \theta_{q} \sin \varphi_{q} \cos \theta\right)\left(-\sin \theta_{q} \sin \theta \cos \varphi+\cos \theta_{q} \cos \theta\right) d \varphi \\
& =\int_{0}^{\pi} \sin \theta d \theta \int_{0}^{2 \pi} \exp (i q a \cos \theta) \cos \theta_{q} \sin \varphi_{q} \cos \varphi_{q} \cos \theta \\
& \times\left(\cos ^{2} \theta_{q} \sin ^{2} \theta \cos ^{2} \varphi+\sin ^{2} \theta_{q} \cos ^{2} \theta\right. \\
& \left.-2 \sin ^{2} \theta_{q} \sin ^{2} \theta \cos ^{2} \varphi-\sin ^{2} \theta \sin ^{2} \varphi\right) d \varphi \\
& =2 \pi i \cos \theta_{q} \sin \varphi_{q} \cos \varphi_{q}\left(\left(-\cos ^{2} \theta_{q}+2 \sin ^{2} \theta_{q}+1\right)\right. \\
& \left.\times\left(j_{0}^{(1)}(q a)+j_{0}^{(3)}(q a)\right)+2 \sin ^{2} \theta_{q} j_{0}^{(3)}(q a)\right) \\
& =2 \pi i \sin ^{2} \theta_{q} \cos \theta_{q} \sin \varphi_{q} \cos \varphi_{q}\left(3 j_{0}^{(1)}(q a)+5 j_{0}^{(3)}(q a)\right) \\
& =2 \pi i v_{x} v_{y} v_{z}\left(3 j_{0}^{(1)}(q a)+5 j_{0}^{(3)}(q a)\right) \text {. }
\end{aligned}
$$

Кроме того, необходимо рассмотреть еще два случая $i=z, j=z, k=z$ и $i=z, j=z, k=x$ :

$$
\begin{aligned}
& \int_{4 \pi} \exp (i \mathbf{q x})\left(n_{z}\right)^{3} d \Omega_{\mathbf{x}^{\prime}}=\int_{0}^{\pi} \sin \theta d \theta \int_{0}^{2 \pi} \exp (i \mathbf{q n} a)\left(\mathbf{n} \mathbf{e}_{z}\right)^{3} d \varphi \\
& =\int_{0}^{\pi} \sin \theta d \theta \int_{0}^{2 \pi} \exp (i q a \cos \theta) \\
& \times\left(-\sin \theta_{q} \sin \theta \cos \varphi+\cos \theta_{q} \cos \theta\right)^{3} d \varphi \\
& =\int_{0}^{\pi} \exp (i q a \cos \theta)\left(3 \pi \sin ^{2} \theta_{q} \cos \theta_{q} \sin ^{2} \theta \cos \theta\right. \\
& \left.+2 \pi \cos ^{3} \theta_{q} \cos { }^{3} \theta\right) \sin \theta d \theta=3 \pi \sin ^{3} \theta_{q} \cos \theta_{q} \\
& \times\left(-2 i\left(j_{0}^{(1)}(q a)+j_{0}^{(3)}(q a)\right)\right)+2 \pi \cos ^{3} \theta_{q} 2 i j_{0}^{(3)}(q a) \\
& =-6 \pi i\left(1-v_{z}^{2}\right) v_{z}\left(j_{0}^{(1)}(q a)+j_{0}^{(3)}(q a)\right)+4 \pi i v_{z}^{3} j_{0}^{(3)}(q a) \\
& =2 \pi i v_{z}^{3}\left(3 j_{0}^{(1)}(q a)+5 j_{0}^{(3)}(q a)\right) \\
& -6 \pi i v_{z}\left(j_{0}^{(1)}(q a)+j_{0}^{(3)}(q a)\right),
\end{aligned}
$$

$\int_{4 \pi} \exp (i \mathbf{q x})\left(n_{z}\right)^{2}\left(n_{x}\right) d \Omega_{\mathbf{x}^{\prime}}=\int_{0}^{\pi} \sin \theta d \theta \int_{0}^{2 \pi} \exp (i \mathbf{q n} a)$

$\times\left(\mathbf{n e}_{z}\right)^{2}\left(\mathbf{n e}_{x}\right) d \varphi=\int_{0}^{\pi} \sin \theta d \theta \int_{0}^{2 \pi} \exp ($ iqa $\cos \theta)$

$\times\left(\left(\sin ^{2} \theta_{q} \sin ^{2} \theta \cos ^{2} \varphi \sin \theta_{q} \cos \varphi_{q} \cos \theta\right)\right.$

$+\left(\cos ^{2} \theta_{q} \cos ^{2} \theta \sin \theta_{q} \cos \varphi_{q} \cos \theta\right)$

$+\left(-2 \sin \theta_{q} \sin \theta \cos \varphi \cos \theta_{q} \cos \theta \cos \theta_{q}\right.$

$\left.\left.\times \cos \varphi_{q} \sin \theta \cos \varphi\right)\right) d \varphi=\pi \sin ^{3} \theta_{q} \cos \varphi_{q}$

$\times \int_{0}^{\pi} \exp ($ iqa $\cos \theta) \sin ^{3} \theta \cos \theta d \theta+2 \pi \cos ^{2} \theta_{q} \sin \theta_{q} \cos \varphi_{q}$

$\times \int_{0}^{\pi} \exp ($ iqa $\cos \theta) \cos ^{3} \theta \sin \theta d \theta-2 \pi \sin \theta_{q} \cos ^{2} \theta_{q} \cos \varphi_{q}$

$\times \int_{0}^{\pi} \exp ($ iqa $\cos \theta) \sin ^{3} \theta \cos \theta d \theta=\pi \sin ^{3} \theta_{q} \cos \varphi_{q}$

$\times\left(-2 i\left(j_{0}^{(1)}(q a)+j_{0}^{(3)}(q a)\right)\right)$

$+2 \pi \cos ^{2} \theta_{q} \sin \theta_{q} \cos \varphi_{q} 2 i j_{0}^{(3)}(q a)$

$-2 \pi \sin \theta_{q} \cos ^{2} \theta_{q} \cos \varphi_{q}\left(-2 i\left(j_{0}^{(1)}(q a)+j_{0}^{(3)}(q a)\right)\right)$

$=-2 \pi i \sin ^{3} \theta_{q} \cos \varphi_{q}\left(j_{0}^{(1)}(q a)+j_{0}^{(3)}(q a)\right)$

$+4 \pi i \cos ^{2} \theta_{q} \sin \theta_{q} \cos \varphi_{q}\left(j_{0}^{(1)}(q a)+2 j_{0}^{(3)}(q a)\right)$

$=-2 \pi i\left(1-v_{z}^{2}\right) v_{x}\left(j_{0}^{(1)}(q a)+j_{0}^{(3)}(q a)\right)$

$+4 \pi i v_{x} v_{z}^{2}\left(j_{0}^{(1)}(q a)+2 j_{0}^{(3)}(q a)\right)$

$=2 \pi i v_{x} v_{z}^{2}\left(3 j_{0}^{(1)}(q a)+5 j_{0}^{(3)}(q a)\right)$

$-2 \pi i v_{x}\left(j_{0}^{(1)}(q a)+j_{0}^{(3)}(q a)\right)$.

Аналогичные выражения будем получать, проводя вычисления для остальных случаев. Обобщая результаты, получаем формулу (22).

\section{Список литературы}

[1] de Beer A.G.F., Roke S. // Phys. Rev. B. 2007. V. 75. N 24. P. 245438. doi 10.1103/PhysRevB.75.245438

[2] Wang H., Yan E.C.Y., Borguet E., Eisenthal K.B. // Chem. Phys. Lett. 1996. V. 259. N 1-2. P. 15. doi 10.1016/00092614(96)00707-5 
[3] Yang N., Angerer W.E., Yodh A.G. // Phys. Rev. Lett. 2001. V. 87. N 10. P. 103902. doi 10.1103/PhysRevLett.87.103902

[4] Jen S.H., Dai H.L. // J. Phys. Chem. B. 2006. V. 110. N 46. P. 23000. doi 10.1021/jp0644762

[5] Jen S.-H., Gonella G., Dai H.-L. // J. Phys. Chem. A. 2009. V. 113. N 6. P. 4758. doi 10.1021/jp9009959

[6] Viarbitskaya S., Kapshai V., van der Meulen P., Hansson T. // Phys. Rev. A. 2010. V. 81. N 5. P. 053850. doi 10.1103/PhysRevA.81.053850

[7] Shan J., Dadap J.I., Stiopkin I., Reider G.A., Heinz T.F. // Phys. Rev. A. 2006. V. 73. N 2. P. 023819. doi 10.1103/PhysRevA.73.023819

[8] Roke S., Bonn M., Petukhov A.V. // Phys. Rev. B. 2004. V. 70. N 11. P. 115106. doi 10.1103/PhysRevB.70.115106

[9] de Beer A.G.F., de Aguiar H.B., Nijsen J.F.W., Roke S. // Phys. Rev. Lett. 2009. V. 102. N 9. P. 095502. doi 10.1103/PhysRevLett.102.095502

[10] Subir M., Liu J., Eisenthal K.B. // J. Phys. Chem. C. 2008. V. 112. N 40. P. 15809. doi 10.1021/jp8047168

[11] de Beer A.G.F., Roke S. // J. Chem. Phys. 2010. V. 132. N 23. P. 234702. doi 10.1063/1.3429969

[12] Liu J., Subir M., Nguyen K., Eisenthal K.B. // J. Phys. Chem. B. 2008. V. 112. N 48. P. 15263-15266. doi $10.1021 / \mathrm{jp} 806690 \mathrm{z}$

[13] Strader M.L., de Aguiar H.B., de Beer A.G.F., Roke S. // Soft Matter. 2011. V. 7. N 10. P. 4959-4963. doi $10.1039 /$ COSM01358G

[14] Jackson J.D. Classical Electrodynamics. N.Y.: Wiley \& Sons, 1998. 832 р.; Джсексон Д.Д. Классическая электродинамика. М.: Мир, 1965. 702 с.

[15] Шамына А.А., Капшай В.Н. // Опт. и спектр. 2018. Т. 124. № 1. C. 105-121. doi 10.21883/OS.2018.01.45366.176-17 Shamyna A.A., Kapshai V.N. // Opt. Spectrosc. 2018. V. 124. N 1. P. 103-120. doi 10.1134/S0030400X18010198;

[16] Капшай В.Н., Шамына А.А. // Опт. и спектр. 2017. T. 123. № 3. C. 416-429. doi 10.7868/S003040341709015X Kapshai V.N., Shamyna A.A. // Opt. Spectrosc. 2017. V. 123. N 3. P. 440-453. doi 10.1134/S0030400X17090144;

[17] de Beer A.G.F., Roke S. // Phys. Rev. B. 2009. V. 79. N 15. P. 155420. doi 10.1103/PhysRevB.79.155420

[18] de Beer A.G.F., Roke S., Dadap J.I. // J. Opt. Sos. Am. B. 2011. V. 28. N 6. P. 1374-1384. doi 10.1103/PhysRevB.79.155420 